\title{
Low-Molecular-Weight Polyethylenimine Enhanced Gene Transfer by Cationic Cholesterol-Based Nanoparticle Vector
}

\author{
Yoshiyuki Hattori* and Yoshie MaItani \\ Institute of Medicinal Chemistry, Hoshi University; 2-4-41 Ebara, Shinagawa-ku, Tokyo 142-8501, Japan. \\ Received May 8, 2007; accepted June 25, 2007; published online June 26, 2007
}

\begin{abstract}
Both polyethylenimine (PEI) polymers and cationic nanoparticles have been widely used for non-viral DNA transfection. Previously, we reported that cationic nanoparticles composed of cholesteryl-3 $\beta$-carboxyamidoethylene- $N$-hydroxyethylamine and Tween 80 (NP-OH) could deliver plasmid DNA (pDNA) with high transfection efficiency. To increase the transfection activity of NP-OH, we investigated the potential synergism of PEI and NPOH for the transfection of DNA into human prostate tumor PC-3, human cervices tumor Hela, and human lung adenocarcinoma A549 cells. The transfection efficiency with low-molecular PEI (MW 600) was low, but that with a combination of NP-OH and PEI was higher than with NP-OH alone, being comparable to commercially available lipofectamine 2000 and lipofectamine LTX, with very low cytotoxicity. Low-molecular weight PEI could not compact pDNA in size, but rather might help to dissociate pDNA from the complex and release pDNA from the endosome to cytoplasm by the proton sponge effect. Therefore, the combination of cationic cholesterol-based nanoparticles and a low-molecular PEI has potential as a non-viral DNA vector for gene delivery.
\end{abstract}

Key words cationic nanoparticle; gene delivery; transfection; cholesteryl-3 $\beta$-carboxyamidoethylene- $N$-hydroxyethylamine; polyethylenimine; nanoplex; PC-3 cell

Gene delivery has become an increasingly important strategy for treating a variety of human diseases, including cancer. $^{1)}$ It is important to develop gene delivery vectors with strong transfection activity and low toxicity for applications in vivo. Many different cationic lipids have been synthesized for delivering genes into cells. The use of cationic cholesterol derivatives is justified by their high transfection activity and low toxicity. ${ }^{2,3}$ Cholesteryl-3 $\beta$-carboxyamidoethylene$N$-hydroxyethylamine ( $\mathrm{OH}-\mathrm{Chol})$, having a hydroxyethyl group at the amino terminal, is a cationic cholesterol derivative with good transfection efficiency for plasmid DNA (pDNA) delivery. ${ }^{2)}$

Polyethylenimines (PEIs) are a large group of non-viral vectors that have been shown to be capable of delivering pDNA into various cell lines. ${ }^{4)} \mathrm{PEI} / \mathrm{pDNA}$ complexes (polyplex) are taken up by a variety of cells via endocytosis, enter the endosomal compartment, and are finally released due to their buffer capacity via the so-called "proton sponge mechanism".5) PEI has the ability to capture protons that are pumped into endosomes. This is followed by a passive influx of chloride ions into endosomes and subsequent osmotic swelling and disruption of the endosomes. This permits the escape of endocytosed DNA. ${ }^{5,6)}$ The transfection efficiency of PEI increases with increasing molecular weight. The low-molecular weight PEIs $(600,1200,1800)$ are virtually ineffective. ${ }^{7)}$ The smallest PEI that has been used in gene transfection has been $11900{ }^{8)}$ Large PEIs, such as PEI 25K (average MW 25000) ${ }^{9-12}$ and PEI 80K (average MW $80000),{ }^{5,9,11}$ have been used successfully in transfection studies. PEIs with a mean molecular weight of less than 2000 are inactive as transfection agents, but can be used in combination with cationic liposomes. ${ }^{13,14)}$

Cationic liposomes are widely used as gene transfection reagents, and bind electrostatically to negatively charged DNA to form complexes. These lipoplexes enter the cells via endocytosis. After endocytosis, the pDNA must escape from the endosomes into the cytoplasm. PEI is able to enhance gene transfer by helping to disrupt the endosomal membrane. ${ }^{4)}$
The use of low-molecular weight PEIs (MW 700, 2000) with liposomes such as dioleoyloxypropyl trimethylammonium methylsulfate (DOTAP) liposome, lipofectamine and polycationic liposome Dosper resulted in a synergistic increase in the transfection efficiency in rat smooth muscle or rat glioma C6 cells, but that with cationic dendrimers (Superfect) did not. ${ }^{13,15)}$ Among them, the combination of Dosper liposome and low-molecular PEI caused the most effective transfection synergism. However, it was not clear whether cationic nanoparticles could enhance transfection efficiency in combination with PEI.

We previously reported that cationic nanoparticles composed of OH-Chol and Tween $80(\mathrm{NP}-\mathrm{OH})$ could deliver pDNA with high transfection efficiency when the nanoparticle/pDNA complex (nanoplex) was formed in a $50 \mathrm{~mm} \mathrm{NaCl}$ solution. ${ }^{16,17)}$ In this study, to increase the transfection efficiency of NP-OH, we evaluated the potential synergism of PEI of low- (average MW 600, 1800) or high- (average MW $10000 ; 10 \mathrm{~K}$ ) molecular weight and NP-OH for pDNA transfection. The transfection resulted in efficient DNA transfer in human prostate tumor PC-3, human cervices tumor Hela, and human lung adenocarcinoma A549 cells as a ternary complex with low-molecular weight PEI 600, NP-OH, and pDNA.

\section{MATERIALS AND METHODS}

Preparation of pDNA The plasmid pCMV-Gluc control encoding secretable Gaussia luciferase (Gluc) under the control of the CMV promoter was purchased from New England Biolabs (MA, U.S.A.). A protein-free preparation of the plasmid was purified following alkaline lysis using maxiprep columns (Qiagen, Hilden, Germany).

Preparation of Nanoparticles, Nanoplexes, and Ternary Complexes The synthesis of $\mathrm{OH}-\mathrm{Chol}$ was done as previously described. ${ }^{16)} \mathrm{NP}-\mathrm{OH}$ formulation consisted of $1 \mathrm{mg} / \mathrm{ml}$ of OH-Chol as a cationic lipid and $5 \mathrm{~mol} \%$ of Tween 80 (NOF Co., Ltd., Tokyo, Japan). NP-OH nanoparticles were 
prepared with lipids (e.g. OH-Chol: Tween $80=10: 1.3$, weight $(\mathrm{mg})$ ) in $10 \mathrm{ml}$ of water by the modified ethanol injection method as previously described. ${ }^{16)}$

The charge ratio $(+/-)$ of NP-OH/pDNA is expressed as the nitrogen of cationic lipid/DNA phosphate ratio. The NP$\mathrm{OH} / \mathrm{pDNA}$ complex (nanoplex) was formed by the addition of $\mathrm{NP}-\mathrm{OH}$ to $2 \mu \mathrm{g}$ of pDNA in water or $50 \mu \mathrm{l}$ of $50 \mathrm{~mm} \mathrm{NaCl}$ solution with gentle shaking and leaving at room temperature for $10 \mathrm{~min}$.

For preparation of the NP-OH/PEI/pDNA complex (ternary complex), we used 3 kinds of branched PEIs (MW 600, 1800, 10000 (10K), Wako Chemicals, Osaka, Japan). The charge ratio $(+/-)$ of $\mathrm{PEI} / \mathrm{pDNA}$ is expressed as the PEI amine nitrogen/DNA phosphate ratio. PEIs were used with charge ratios $(+/-)$ of 1,2 and 3 , and $\mathrm{NP}-\mathrm{OH}$ nanoparticles had cationic lipid/DNA ratios of between 1 and 3. Briefly, to form $\mathrm{PEI} / \mathrm{pDNA}$ complexes (polyplexes), pDNA was combined with the PEI solution to achieve charge ratios $(+/-)$ of 1,2 , and 3 in water or $5-50 \mathrm{~mm} \mathrm{NaCl}$ solution and incubated for $10 \mathrm{~min}$. Ternary complexes were formed by the addition of nanoparticles to the polyplex solution at the NP$\mathrm{OH} / \mathrm{DNA}$ ratios mentioned above, and left at room temperature for another $10 \mathrm{~min}$. The particle size distributions and $\zeta$ potentials were measured with an ELS-Z2 (Otsuka Electronics Co., Ltd., Osaka, Japan), at $25^{\circ} \mathrm{C}$ after diluting the dispersion to an appropriate volume with water. The average size and $\zeta$-potential of NP-OH were approximately 120 $130 \mathrm{~nm}$ and $+45-50 \mathrm{mV}$, respectively.

Cell Culture PC-3 cells were supplied by the Cell Resource Center for Biomedical Research, Tohoku University (Miyagi, Japan). Hela cells were obtained from the European Collection of Cell Culture (Wiltshire, U.K.). A549 cells were a gift from Oncotherapy Science (Tokyo, Japan). The cells were grown in RPMI-1640 medium (Invitrogen Corp., Carlsbad, CA, U.S.A.) supplemented with $10 \%$ heat-inactivated fetal bovine serum (FBS) (Invitrogen Corp., Carlsbad, CA, U.S.A.) and kanamycin $(100 \mu \mathrm{g} / \mathrm{ml})$ at $37^{\circ} \mathrm{C}$ in a $5 \% \mathrm{CO}_{2}$ humidified atmosphere.

Secreted Gaussia Luciferase Assay PC-3, Hela, and A549 cells were plated into 96-well culture dishes. For transfection, nanoplexes, polyplexes and ternary complexes were diluted in $1 \mathrm{ml}$ of medium supplemented with $10 \% \mathrm{FBS}$ and then $100 \mu \mathrm{l}$ of the mixture were incubated with the cells for 24 and $48 \mathrm{~h}$. Lipofectamine 2000 and lipofectemine LTX lipoplexes (Invitrogen Corp.) were prepared according to the manufacturer's protocol. Activity of secreted Gaussia luciferase in medium was measured as counts per second (cps)/culture medium $(\mu \mathrm{l})$ using a Gaussia Luciferase Assay Kit (New England BioLabs, Inc., MA, U.S.A.) according to the manufacturer's directions.

Gel Retardation Assay One microgram of pDNA was mixed with aliquots of PEI $600,1800,10 \mathrm{~K}$, and NP-OH ( 1 to 3 charge equivalents of cationic polymer or lipid) in $50 \mathrm{~mm}$ $\mathrm{NaCl}$ solution. After a 10-min incubation, the polyplexes and nanoplexes were analyzed by $1.5 \%$ agarose gel electrophoresis in Tris-Borate-EDTA (TBE) buffer and visualized by ethidium bromide staining as previously described. ${ }^{16)}$

Cytotoxicity PC-3, Hela, and A549 cells were plated into 96-well culture dishes. Cytotoxicity upon transfection using nanoplexes, ternary complexes, lipofectamine 2000 , or lipofectamine LTX was evaluated with a cell proliferation assay kit (Dojindo, Kumamoto, Japan). Nanoplexes and ternary complexes were prepared in $50 \mathrm{~mm} \mathrm{NaCl}$ solution as described above, and transfected into the cells in medium containing $10 \% \mathrm{FBS}$. After $48 \mathrm{~h}$ of incubation, the medium was removed, and the cells were treated with a WST-8 (2-(2methoxy-4-nitrophenyl)-3-(4-nitrophenyl)-5-(2,4-disulfophenyl)- $2 H$-tetrazolium, monosodium salt) solution $(10 \mu \mathrm{l})$ in medium containing serum $(100 \mu \mathrm{l})$ for $30 \mathrm{~min}$. Cell viability was expressed relative to the absorbance at $450 \mathrm{~nm}$ of untransfected cells.

Statistical Analysis Multiple measurement comparisons were performed with an analysis of variance followed by a one-way analysis of variance on ranks with a post-hoc Tukey-Kramer test. A $p$ value of 0.05 or less was considered significant.

\section{RESULTS}

PEI Polyplex-Mediated Gene Transfer First, the transfection efficiency of the PEI polyplexes formed with PEI 600,1800 , and $10 \mathrm{~K}$ was evaluated in PC-3 cells. Among the PEI polyplexes formed in water, the PEI 10K polyplex showed higher transfection activity than the PEI 600 and 1800 polyplexes, and its luciferase activity increased in parallel with the increasing charge ratio $(+/-)$ (Fig. 1A). However, the transfection efficiency was significantly lower than that of commercial products. This finding corresponded to the finding that PEIs of low-molecular weights produced low transfection efficiency. ${ }^{7)}$ The presence of $\mathrm{NaCl}$ in the polyplex did not increase transfection efficiency (Fig. 1B).
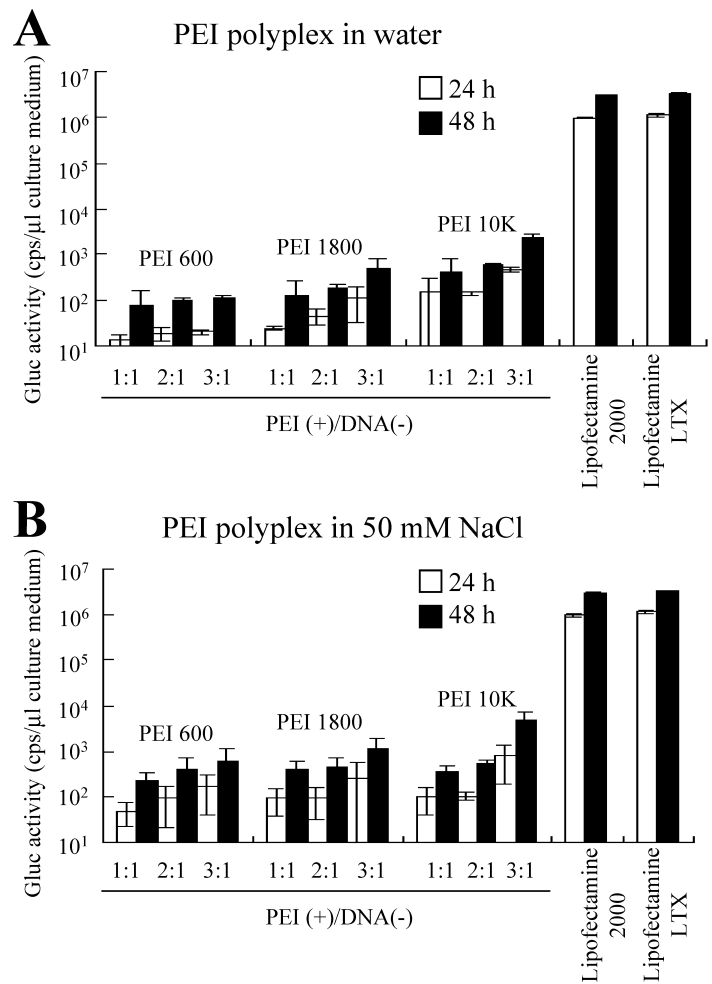

Fig. 1. Effect of Charge Ratio (+/-) of PEI/pDNA Polyplex on Transfection in PC-3 Cells

Polyplexes were formed in water (A) or a $50 \mathrm{~mm} \mathrm{NaCl}$ solution (B) at charge ratios $(+/-)$ of PEIs to pDNA of $1 / 1,2 / 1$ and $3 / 1$. Each column represents the mean \pm S.D. $(n=3)$ 

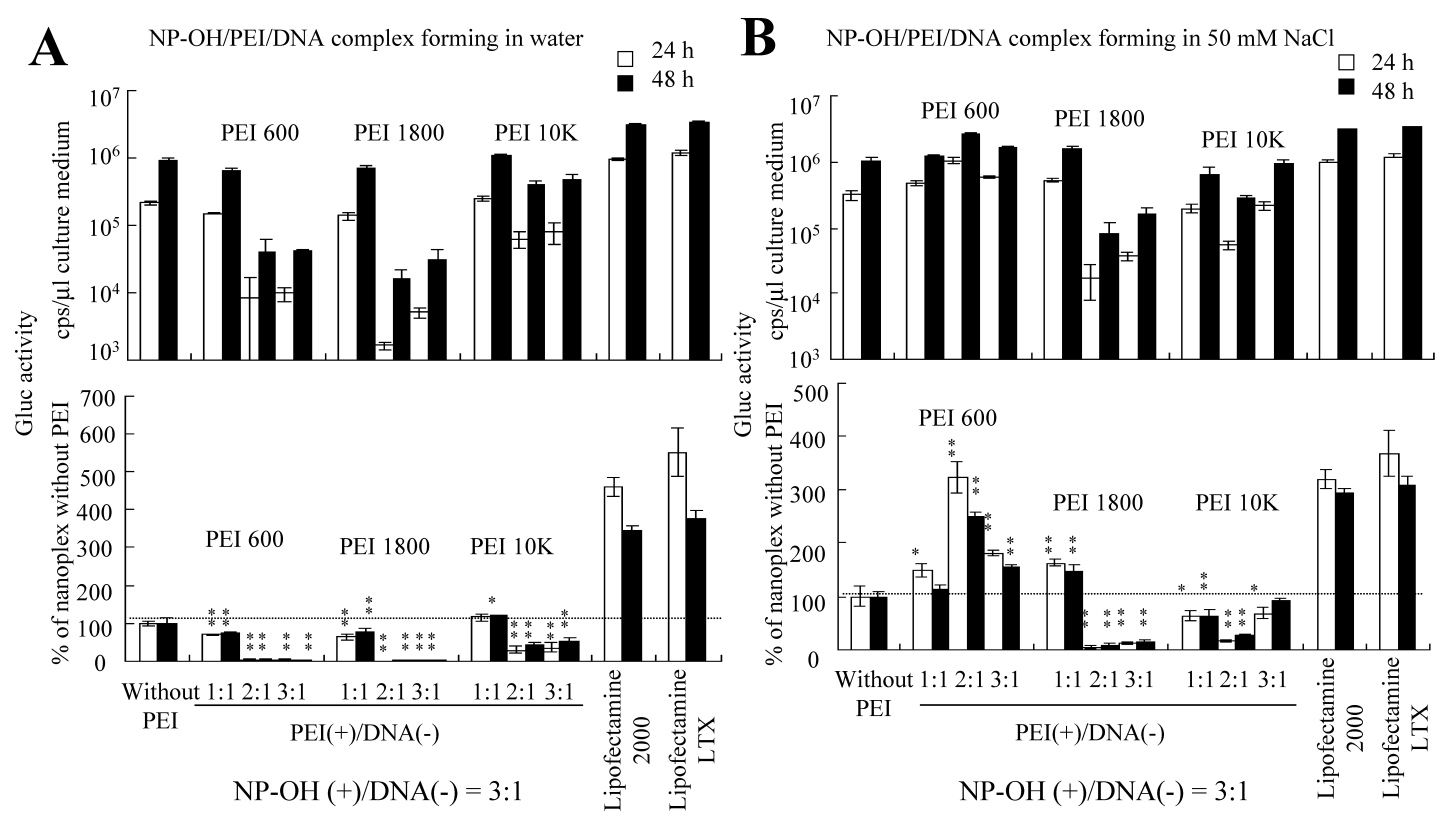

Fig. 2. Effect of Charge Ratio (+/-) of PEI/pDNA in Ternary Complex on Transfection in PC-3 Cells

Using polyplexes formed in water (A) or a $50 \mathrm{~mm} \mathrm{NaCl}$ solution (B) at charge ratios (+/-) of 1/1, 2/1, and 3/1, the ternary complex was formed at a charge ratio (+/-) of 3/1. $* * p<0.01$ and $* p<0.05$, compared with a ternary complex without PEI. Each column represents the mean \pm S.D. $(n=3)$.
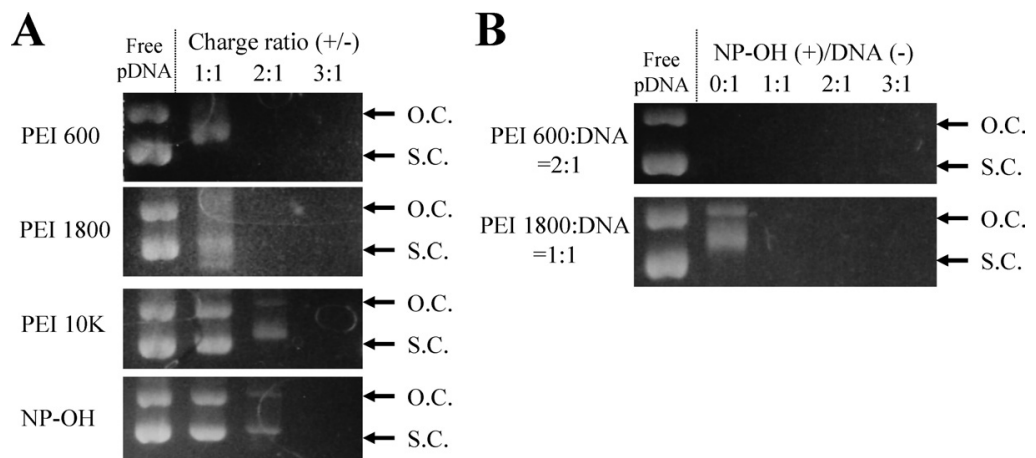

Fig. 3. Association of pDNA with PEI and NP-OH in $50 \mathrm{~mm} \mathrm{NaCl}$ at Various Charge Ratios (+/-) Was Analyzed Using $1.5 \%$ Agarose Gel Electrophoresis

One microgram of pDNA was mixed with aliquots of PEI $600,1800,10 \mathrm{~K}$ or NP-OH at various charge ratios $(\mathrm{A})$. Ternary complexes at charge ratios $(+/-)$ of $2 / 1$ for PEI 600 and 1/1 for PEI 1800 were formed at various charge ratios $(+/-)$ of NP-OH (B). O.C. indicates open circular plasmid; S.C. indicates supercoiled plasmid.

Effect of PEI/pDNA Ratio in Ternary Complex on Transfection The effect on transfection efficiency of different charge ratios $(+/-)$ of the $\mathrm{PEI} / \mathrm{pDNA}$ in $\mathrm{NP}-\mathrm{OH}$ ternary complexes was studied (Fig. 2). Charge ratio $(+/-)$ of $\mathrm{NP}-\mathrm{OH} / \mathrm{pDNA}$ ratio of $3 / 1$ was chosen, since this had been effective when used alone. ${ }^{16,17)}$ Ternary complexes prepared in water were all ineffective in the cells (Fig. 2A). Gluc activity in the culture medium decreased with the increasing charge ratio $(+/-)$ of $\mathrm{PEI} / \mathrm{pDNA}$. However, when the ternary complex was prepared in $50 \mathrm{~mm} \mathrm{NaCl}$, PEI 600 at a charge ratio $(+/-)$ of $2 / 1$ and PEI 1800 at a charge ratio $(+/-)$ of $1 / 1$ increased transfection efficiency compared with the complex without PEI, i.e., the nanoplex (Fig. 2B). This indicated that a combination of NP-OH and PEI 600 or 1800 could increase transfection when the ternary complex was formed in the presence of a $\mathrm{NaCl}$ solution. Therefore, in subsequent experiments, we decided to use PEI 600 at a charge ratio $(+/-)$ of $2 / 1$ and PEI 1800 at a charge ratio $(+/-)$ of $1 / 1$ as optimal $\mathrm{PEI} / \mathrm{pDNA}$ ratios.

Gel Retardation Assay The association of pDNA with
PEI and/or NP-OH was monitored by gel retardation electrophoresis. The migration pattern of pDNA in the polyplex and nanoplex changed when the pDNA was mixed with PEI or $\mathrm{NP}-\mathrm{OH}$ at charge ratios $(+/-)$ from 1 to 3 in $50 \mathrm{~mm} \mathrm{NaCl}$ solution. Beyond a charge ratio $(+/-)$ of $2 / 1$ in PEI 600 and 1800 polyplexes, no migration was observed (Fig. 3A). Beyond a charge ratio $(+/-)$ of $3 / 1$ in the PEI $10 \mathrm{~K}$ polyplex and NP-OH nanoplex, no migration was observed (Fig. 3A). These results indicated that a complete complex was formed above a charge ratio $(+/-)$ of $2 / 1$ in PEI 600 and 1800, and of $3 / 1$ in PEI $10 \mathrm{~K}$ and NP-OH. Furthermore, in ternary complexes at charge ratios $(+/-)$ of $2 / 1$ for PEI 600 and $1 / 1$ for PEI 1800, no migration was observed beyond a charge ratio $(+/-)$ of NP-OH/pDNA of $1 / 1$ (Fig. 3B), indicating that the ternary complexes of PEI 600 and 1800 used in Fig. 2B were completely formed complexes with pDNA. $\zeta$-Potentials were about +3 and $-34 \mathrm{mV}$ when the polyplexes were formed in $50 \mathrm{~mm} \mathrm{NaCl}$ at a charge ratio $(+/-)$ of $2 / 1$ for PEI 600 and $1 / 1$ for PEI 1800, respectively, and increased to +25 and $+35 \mathrm{mV}$ after the formation of ternary complexes at a charge 
ratio $(+/-)$ of NP-OH/pDNA of $3 / 1$ (data not shown). This finding also suggested that the pDNA partially neutralized by PEI could be associated with NP-OH.

The Role of NP-OH/pDNA Ratios in Delivery Next, to increase transfection efficiency, we studied the effect of NP$\mathrm{OH} / \mathrm{pDNA}$ ratios in combination with PEI 600 or 1800 . We

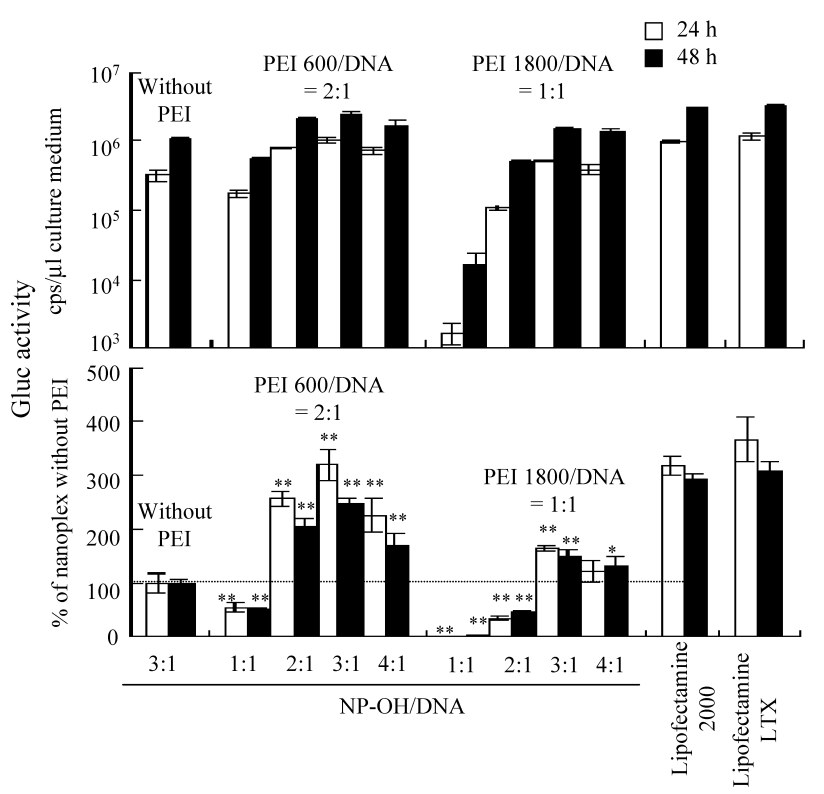

Fig. 4. Effect of Charge Ratio (+/-) of NP/pDNA in Ternary Complex on Transfection in PC-3 Cells

Using polyplexes formed in a $50 \mathrm{~mm} \mathrm{NaCl}$ solution at charge ratios $(+/-)$ of $2 / 1$ for PEI 600 and $1 / 1$ for PEI 1800, the ternary complex was formed at various charge ratios $(+/-)$ from $1 / 1$ to $4 / 1$. Each column represents the mean \pm S.D. $(n=4) . * * p<0.01$ and $* p<0.05$, compared with a ternary complex without PEI. used a charge ratio $(+/-)$ of PEI/pDNA of $2 / 1$ for PEI 600 and of 1/1 for PEI 1800 in ternary complexes from the result in Fig. 2B. At a charge ratio $(+/-)$ of NP-OH/pDNA of 3/1, the transfection efficiencies were highest in both PEI 600 and 1800 (Fig. 4). Thereafter, for the optimal PEI 600 and 1800 ternary complexes, we used $3 / 1$ as the charge ratio $(+/-)$ of $\mathrm{NP}-\mathrm{OH} / \mathrm{pDNA}$. Comparison of the molecular weight of PEI in combination with NP-OH revealed that PEI 600 was more effective than PEI 1800. This finding corresponded with the notion that a low-molecular weight PEI could be used successfully for gene delivery when combined with cationic liposomes. ${ }^{14)}$

Effect of Sodium Chloride on the Size of the Ternary Complex We compared the physicochemical properties of ternary complexes at a charge ratio $(+/-)$ of $\mathrm{NP}-\mathrm{OH} / \mathrm{pDNA}$ of $3 / 1$ formed in the presence of various concentrations of $\mathrm{NaCl}$ solution. The NP-OH nanoplex formed in water and the $50 \mathrm{~mm} \mathrm{NaCl}$ solution was about 290 and $820 \mathrm{~nm}$ in size, respectively. The PEI 600 polyplex formed in the $50 \mathrm{~mm}$ $\mathrm{NaCl}$ solution at a charge ratio $(+/-)$ of $2 / 1$ was about $830 \mathrm{~nm}$. The ternary complex formed in water or the $50 \mathrm{~mm}$ $\mathrm{NaCl}$ solution was about $1500 \mathrm{~nm}$ in size (Fig. 5A). A significant difference was not observed in the sizes of the ternary complexes formed between 0 and $100 \mathrm{~mm} \mathrm{NaCl}$.

Effect of Sodium Chloride on the Transfection Efficiency of the Ternary Complex We investigated the effect of sodium chloride on the transfection efficiency of ternary complexes in PC-3 cells. The presence of $\mathrm{NaCl}$ in the ternary complex increased transfection efficiency in parallel with the increasing concentration of $\mathrm{NaCl}$, and a saturated transfection efficiency was observed at $50 \mathrm{~mm} \mathrm{NaCl}$ (Fig. 5B). The observed transfection efficiency 24 and $48 \mathrm{~h}$ after transfec-

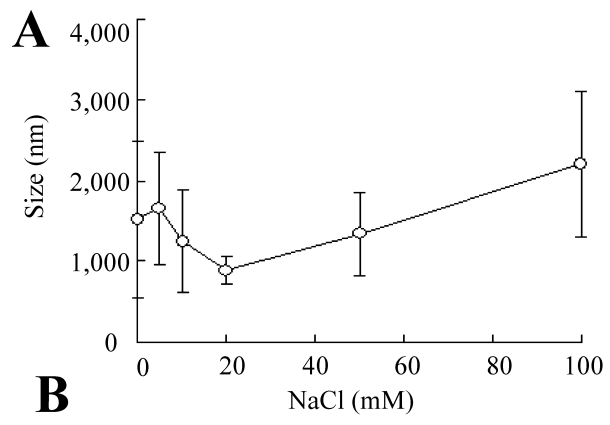

C
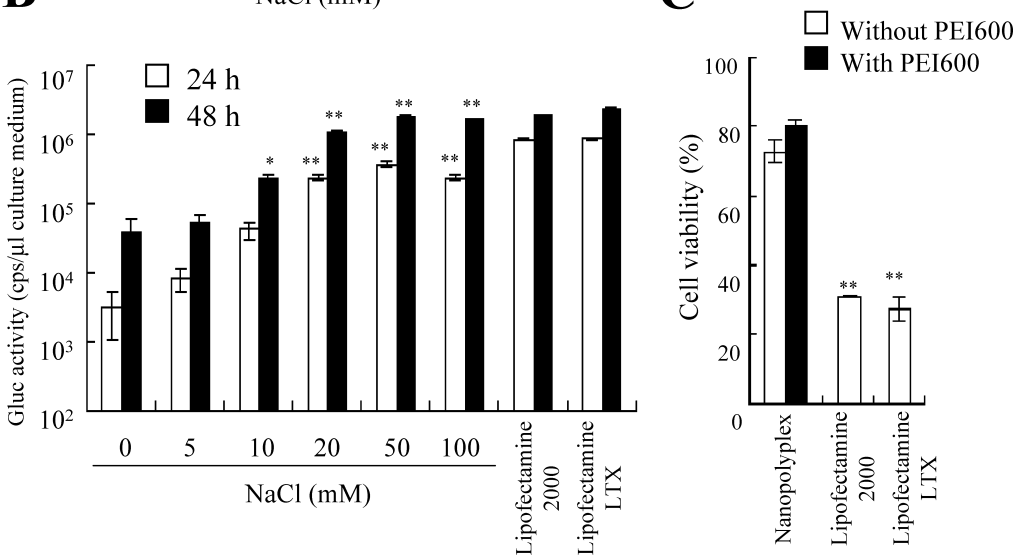

Fig. 5. Effect of Sodium Chloride in the NP-OH Ternary Complex Using Polyplexes Formed with Various Concentrations of NaCl at a Charge Ratio $(+/-)$ of $2 / 1$ for PEI 600 on Size (A) and Transfection in PC-3 Cells (B)

Each value represents the mean \pm S.D. $(n=4) . * p<0.05$ and $* * p<0.01$, compared with the ternary complex formed in water. Comparison of cytotoxicity in PC-3 cells $48 \mathrm{~h}$ after transfection with the ternary complex formed in $50 \mathrm{~mm} \mathrm{NaCl}$ (Fig. $5 \mathrm{~B}$ ) (C). Each column represents the mean \pm S.D. $(n=4)$. 


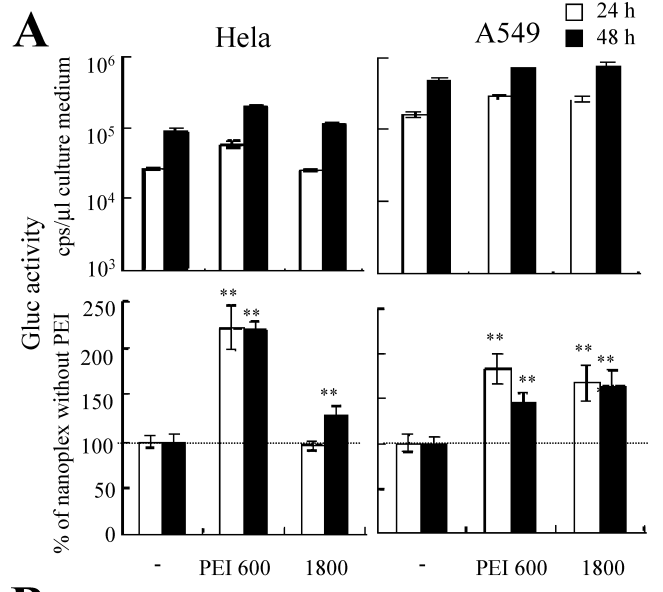

B

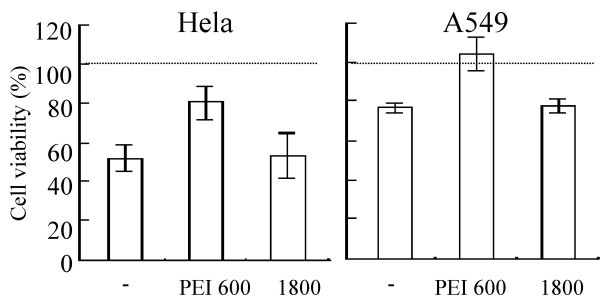

Fig. 6. Comparison of Transfection Levels (A) and Cytotoxicity (B) in Hela and A549 Cells by Ternary Complex $48 \mathrm{~h}$ after Transfection

Using polyplexes formed in a $50 \mathrm{~mm} \mathrm{NaCl}$ solution at charge ratios $(+/-)$ of $2 / 1$ for PEI 600 and $1 / 1$ for PEI 1800, the ternary complex was formed at a charge ratio $(+/-)$ of $3 / 1 . * * p<0.01$, compared with the nanoplex formed in water. Each column represents the mean \pm S.D. $(n=4)$

tion was comparable to those for lipofectamine 2000 and lipofectamine LTX. Therefore, we decided to use $50 \mathrm{~mm}$ $\mathrm{NaCl}$ when forming nanoplexes as an optimal concentration of $\mathrm{NaCl}$.

Cytotoxicity after Transfection of Ternary Complexes Next, we examined the cytotoxicity of the ternary complexes $48 \mathrm{~h}$ after transfection. We compared the cytotoxicity of the ternary complex, the lipofectamine 2000 lipoplex, or the lipofectamine LTX lipoplex in PC-3 cells. The lipofectamine 2000 and lipofectamine LTX lipoplexes exhibited significant toxicity (about 30\% cell viability) (Fig. 5C). In contrast, ternary complexes did not actually exhibit cytotoxicity $(80 \%$ cell viability). These findings indicated that PEI 600 could increase the transfection efficiency of NP-OH without cytotoxicity.

Comparison of Transfection Activity and Cytotoxicity in Hela and A549 Cells Finally, we investigated the transfection efficiency and cytotoxicity of PEI 600 and 1800 ternary complexes in other cells. In Hela and A549 cells, PEI 600 or 1800 combined with NP-OH, increased transfection 1.5-2-fold compared to the nanoplex alone (Fig. 6A). The cytotoxicity of the PEI 600 ternary complex was decreased compared with that of the nanoplex and PEI 1800 ternary complex (Fig. 6B). A lower molecular weight PEI was more effective in increasing transfection activity by $\mathrm{NP}-\mathrm{OH}$ in Hela and A549 cells as well as PC-3 cells. These findings suggested that the combination of NP-OH and PEI 600 has great potential for efficient DNA transfection with low cytotoxicity.

\section{DISCUSSION}

In this study, we investigated the potential synergism of a PEI and NP-OH for the transfection of DNA into PC-3, Hela, and A549 cells, and demonstrated that NP-OH ternary complexes made using a low-molecular weigh PEI had higher levels of transfection efficiency than nanoplexes although the PEI polyplex alone did not exhibit effective gene transfection.

The size of a nanoplex is known to affect its transfection efficiency. ${ }^{18-20)}$ Increased transfection activity was observed when the NP-OH ternary complex was formed at concentrations of $\mathrm{NaCl}$ above $50 \mathrm{~mm}$, even though the size was not significantly different with that formed in water (Figs. 5A, B). We previously reported that a marked dissociation of DNA from NP-OH nanoplexes was observed at low $\mathrm{pH}$ when the nanoplex of DNA was formed in $\mathrm{NaCl}$ solution. ${ }^{16)}$ Since the presence of $\mathrm{NaCl}$ did not increase significantly the size of the $\mathrm{NP}-\mathrm{OH}$ ternary complex, the $\mathrm{NaCl}$ solution might influence the extent to which DNA dissociates from the NP-OH/PEI ternary complex. The presence of $\mathrm{NaCl}$ in forming ternary complex can weaken the association with DNA by neutralization of cationic charge on PEI or on the surface of NP$\mathrm{OH}$. The reason for the high transfection efficiency of the ternary complexes produced in the $\mathrm{NaCl}$ solution may be their instability in the endosome, resulting in the release of DNA from ternary complexes and the translocation of DNA into the cytoplasm.

The mechanistic pathway for gene transfection includes the compaction of the extended pDNA chain, known as DNA condensation. ${ }^{21)}$ Multivalent cations such as polyamines (spermidine, spermine), PEI and peptides (poly-L-lysine) are known to provoke the condensation of DNA to nanoparticles. ${ }^{223)}$ Regarding pDNA compaction with PEI, it has been reported that PEI $25 \mathrm{~K}$ could form small polyplexes $(100$ $250 \mathrm{~nm}$ ) with pDNA, but PEI 2000 could not (greater than $3 \mu \mathrm{m}),{ }^{15)}$ indicating that PEIs with a large molecular weight could compact pDNA. PEIs of more than 11900 in molecular weight induced high gene transfection. $\left.{ }^{8}\right)$ Therefore, the PEI used in this study could neither compact pDNA nor deliver pDNA into cells. The PEI of NP-OH ternary complex may act simultaneously in the release of DNA from endosomes, thus enhancing gene transfer. This finding corresponded to reports that the combination of Dosper liposome plus PEI 700 or 2000 caused the most effective transfection synergism. ${ }^{13-15)}$ With the combination of Dosper liposome and PEI 700, the PEI of the lipopolyplex had no effect on DNA condensation or the complex's size. ${ }^{13,15)}$ The PEI polyplex could protect the pDNA from degradation, or the transport of the PEI polyplex to the nucleus could be more effective than that of naked pDNA. PEI has the ability to more DNA into the nucleus. ${ }^{10)}$ These properties may be more effective with the small molecular PEIs.

When a lipopolyplex with effective transfection activity was formed at charge ratios $(+/-)$ of $2.5 / 1$ for PEI 2000 and $7.5 / 1$ for Dosper liposome in a $150 \mathrm{~mm} \mathrm{NaCl}$ solution, it was about $1500-3000 \mathrm{~nm}$ in size. ${ }^{15)}$ When a ternary complex was formed at charge ratios $(+/-)$ of $2 / 1$ for PEI 600 and $3 / 1$ for $\mathrm{NP}-\mathrm{OH}$ in a $50 \mathrm{~mm} \mathrm{NaCl}$ solution, it was about $1000-1500 \mathrm{~nm}$ in size (Fig. 5A). These findings indicated that the ternary complex was smaller in size and in the 
amount of cationic charge used in transfection than the lipopolyplex. The combination of NP-OH and PEI 600 might be valuable for $\mathrm{pDNA}$ transfection.

We used the WST-8 assay for the cytotoxicity assessment. In PC-3, Hela, and A549 cells, only low toxicity was found on transfection with the PEI600 ternary complex (about 80 $100 \%$ cell viability) (Figs. 5C, 6B). The higher molecular weight of PEIs (PEI 1800) increased cytotoxicity when combined with NP-OH (Fig. 6B). PEIs with low-molecular weight are reported to be less cytotoxic than those with high-molecular weight. ${ }^{8)}$ These findings may explain the rather low toxicity obtained with the PEI 600 ternary complex. For the development of transfection reagents with low cytotoxicity, it is neccessary to have sufficient transfection efficiency with as little cationic lipid as possible. Increasing the charge ratio $(+/-)$ of cationic nanoparticles/pDNA increased not only transfection efficiency but also cytotoxicity by increasing the lipid concentration. Combining NP-OH with PEI 600 allows the use of a smaller amount of NP-OH to obtain sufficient transfection efficiency without an increase of cytotoxicity.

In this study, we demonstrated that the combination of NP$\mathrm{OH}$ and a PEI of low-molecular weight could serve as an efficient vector for DNA transfer, being comparable with commercial reagents. The combination of a PEI polymer and lipid-based nanoparticles is a potential non-viral DNA vector for gene delivery.

Acknowlegements This project was supported in part by a Grant-in-Aid for Scientific Research from the Ministry of Education, Culture, Sports, Science and Technology of Japan.

\section{REFERENCES}

1) Dass C. R., Burton M. A., J. Pharm. Pharmacol., 51, 755-770 (1999).
2) Hasegawa S., Hirashima N., Nakanishi M., Bioorg. Med. Chem. Lett., 12, 1299-1302 (2002).

3) Nakanishi M., Curr. Med. Chem., 10, 1289-1296 (2003).

4) Godbey W. T., Wu K. K., Mikos A. G., J. Control Release, 60, 149160 (1999).

5) Boussif O., Lezoualc'h F., Zanta M. A., Mergny M. D., Scherman D., Demeneix B., Behr J. P., Proc. Natl. Acad. Sci. U.S.A., 92, 7297-7301 (1995).

6) Kichler A., Leborgne C., Coeytaux E., Danos O., J. Gene Med., 3, 135-144 (2001).

7) Godbey W. T., Wu K. K., Mikos A. G., J. Biomed. Mater. Res., 45 268-275 (1999).

8) Fischer D., Bieber T., Li Y., Elsasser H. P., Kissel T., Pharm. Res., 16, 1273-1279 (1999).

9) Abdallah B., Hassan A., Benoist C., Goula D., Behr J. P., Demeneix B. A., Hum. Gene Ther., 7, 1947-1954 (1996).

10) Pollard H., Remy J. S., Loussouarn G., Demolombe S., Behr J. P., Escande D., J. Biol. Chem., 273, 7507-7511 (1998).

11) Boletta A., Benigni A., Lutz J., Remuzzi G., Soria M. R., Monaco L., Hum. Gene Ther., 8, 1243-1251 (1997).

12) Goula D., Benoist C., Mantero S., Merlo G., Levi G., Demeneix B. A., Gene Ther., 5, 1291-1295 (1998).

13) Lampela P., Soininen P., Urtti A., Mannisto P. T., Raasmaja A., Int. J. Pharm., 270, 175-184 (2004).

14) Lampela P., Raisanen J., Mannisto P. T., Yla-Herttuala S., Raasmaja A., J. Gene Med., 4, 205-214 (2002).

15) Lampela P., Elomaa M., Ruponen M., Urtti A., Mannisto P. T., Raasmaja A., J. Control Release, 88, 173-183 (2003).

16) Hattori Y., Kubo H., Higashiyama K., Maitani Y., J. Biomed. Nanotech., 1, 176-184 (2005).

17) Hattori Y., Ding W., Maitani Y., J. Control Release, 120, 122-130 (2007).

18) Almofti M. R., Harashima H., Shinohara Y., Almofti A., Li W., Kiwada H., Mol. Membr. Biol., 20, 35-43 (2003).

19) Turek J., Dubertret C., Jaslin G., Antonakis K., Scherman D., Pitard B., J. Gene Med., 2, 32-40 (2000).

20) Zaric V., Weltin D., Erbacher P., Remy J. S., Behr J. P., Stephan D., J. Gene Med., 6, 176-184 (2004).

21) Bloomfield V. A., Curr. Opin. Struct. Biol., 6, 334-341 (1996).

22) De Smedt S. C., Demeester J., Hennink W. E., Pharm. Res., 17, 113$126(2000)$.

23) Vijayanathan V., Thomas T., Thomas T. J., Biochemistry, 41, 1408514094 (2002). 